ARTIGO ORIGINAL

\title{
Mortalidade em portadores de HIV/AIDS no Pará
}

\author{
Mortality to with HIV/AIDS in Pará \\ Karolaine Galvão Pontes \\ Universidade do Estado do Pará, campus Belém. E-mail: kgalvao808@gmail.com \\ Erica Silva de Souza Matsumura \\ Universidade do Estado do Pará, campus Belém. E-mail: erica.s.souza@terra.com.br \\ Katiane da Costa Cunha \\ Universidade do Estado do Pará, campus Belém. E-mail: katiane.cunha@uepa.br
}

\begin{abstract}
Resumo: Verificar o índice de mortalidade em portadores do vírus da imunodeficiência humana (HIV) e/ou aids no estado do Pará entre os anos de 2007 a 2017. Trata-se de um estudo descritivo, transversal e retrospectivo, por meio da base de dados DATASUS (departamento de informática do sistema único de saúde) na faixa etária de 20 a 59 anos. Como resultados temos, a região metropolitana I apresentou maior prevalência de óbitos, seguida de Carajás. A idade onde se concentrou maior mortalidade foi em adultos de 30 a 39 anos, além de que o estado civil mais comum foram os declarados solteiros. Em relação ao índice de escolaridade, a maioria se concentrou entre 4 a 7 anos de estudo, e no que concerne a cor/raça, a parda foi a mais comum. A respeito da região Metropolitana I apesar de ter quantitativo menor de municípios foi a com maior mortalidade devido a questão populacional, a discrepância entre homens e mulheres se deve ao fato de que eles não procuram centros de saúde com assiduidade, já o tempo de estudo foi relativamente curto devido a problemas escolares/reprovações. Portanto, o HIV ainda é um importante marcador de mortalidade no estado do Pará, e os homens ainda são maioria nesses casos.
\end{abstract}

Palavras-chave: Hiv. Mortalidade. Adultos.

\begin{abstract}
To verify the mortality index in carries of the human deficiency vírus (HIV) and/or aids in the state of Pará between the years 2007 to 2017. This is a study is descriptive, cross-sectionalnand retrospective, using the Datasus (it departamento of the single system). In the age group of 20 to 59 years. As results we have Metropolitana region I had a higher prevalence of deaths, followed by Carajás. The age where the highest mortality was concentrated was in the adults from 30 to 39 years old, in addition to the most common marital status being those declared unmarried. In relation to the level of education, most were concentrated between 4 to 7 years of study and with regard to color/ race, brow skin was more common. About the region Metropolitana region I despite having a smaller number of municipalities, was the one with the highest mortality due to population issues the discrepancy batween men and women id due the fact that they did not look for health centers with assiduity since the study time was relatively short due to school problems/failures. Therefore the HIV is na importante mortality marker in the state of Pará and men are still the majority in cases.
\end{abstract}

Keywords: Hiv. Mortality. Adults. 


\section{INTRODUÇÃO}

A AIDS (Síndrome da Imunodeficiência Adquirida) é considerada mundialmente como um dos principais problemas de saúde pública. Apesar de atingir pessoas de diferentes classes sociais, desde a sua descoberta nos anos de 1980, foi relacionada como uma doença de transmissão sexual propiciada por atividades homossexuais. A aids só foi identificada no Brasil em 1982, quando sete pacientes homo/bissexuais foram diagnosticados com a doença. Com o decorrer dos anos, o seu perfil epidemiológico foi se modificando e alcançou os heterossexuais, brancos, negros, pobres e ricos (LIMA et al.,2017,p.140).

$\mathrm{O}$ perfil da aids nos últimos 10 anos no Brasil, mostrou uma tendência de queda de casos em mulheres e aumento em homens, em 2016 foram 22 casos de aids em homens para cada 10 casos em mulheres (BRASIL, 2017). Passados mais de 30 anos do início da epidemia, o binômio HIV/aids permanece como importante preocupação a nível de saúde pública global, mesmo diante dos avanços nas medidas de prevenção primária e secundária, como inventivo ao uso de preservativos, criação dos Centros de Testagem e Aconselhamento (CTA) para diagnóstico precoce da infecção, e fornecimento gratuito da terapia antirretroviral (TRINDADE, et al., 2019, p. 155).

Com relação à mortalidade por HIV/Aids, observa-se importante redução global $(45 \%$ de 2005 a 2015) (GUIMARÃES et al., 2017). Desde o início da epidemia de aids (1980) até 31 de dezembro de 2018, foram notificados no Brasil 338.905 óbitos tendo o HIV/aids como causa básica (CID10: B20 a B24). A maior proporção desses óbitos ocorreu na região Sudeste $(58,3 \%)$, seguida das regiões Sul $(17,7 \%)$, Nordeste $(13,6 \%)$, Centro-Oeste $(5,3 \%)$ e Norte $(5,1 \%)$. Entre 2008 a 2018 houve uma queda $24,1 \%$ no coeficiente de mortalidade, entretanto a região norte teve um aumento de 26,0\% assim como o nordeste com 2,8\% (BRASIL, 2019).

Em outro artigo que corrobora para os dados acima, demonstra que há importantes diferenças por região. Enquanto para a Região Sudeste há evidente tendência de redução $(b=-0,2354)$, para as Regiões Nordeste e Norte há indicação de aumento da mortalidade (respectivamente, $\mathrm{b}=0,1179$ e $\mathrm{b}=0,2889)$, onde $\mathrm{b}$ é o estudo de carga da doença (GUIMARÃES et al., 2017).

No estado do Pará, segundo a SESPA- Secretaria de Estado de Saúde Pública (2019), 20.547 pessoas estão fazendo tratamento com antirretroviral para HIV e aids. Em 2018, 681 pessoas foram a óbito por aids no estado e, até 30 de junho de 2019, ocorreram mais 312 mortes. As consequências na população estão diretamente ligadas a adesão à terapia antirretroviral, além de implicar na saúde individual, está associada à potencial transmissão da infecção pelo HIV/aids de forma coletiva, e trabalhar em políticas públicas que combatam ao preconceito e estigma a essas pessoas para que elas tenham uma qualidade de vida elevada (Carvalho et al., 2017).

Ao longo do período de 2013 a 2018, verifica-se que, a partir de 2014, houve uma inversão do número de casos de HIV em relação aos de aids. Isto pode demonstrar que há um aumento da detecção de novos casos e que o tratamento é efetivo, diminuindo o número de casos de aids, evitando a progressão para imunodeficiência contribuindo assim para uma menor mortalidade (BRASIL, 2019).

Portanto, cabe ressaltar a importância de se discutir acerca da mortalidade em decorrência da aids no Pará tendo em vista que há poucos trabalhos sobre o perfil epidemiológico dos soropositivos na região o que dificulta medidas profiláticas em grupos de risco. Por conseguinte, este trabalho tem como objetivo esclarecer o índice de mortalidade em portadores de HIV/aids em adultos no estado do Pará no período de 2007 a 2017, assim como associações com as variáveis: sexo, escolaridade, estado civil e cor/raça.

\section{MÉTODO}

Trata-se de um estudo retrospectivo, transversal e descritivo, datado no período de 2007 a 2017. Para a execução do trabalho foi feita a coleta de dados utilizando o Datasus (departamento de informática do Sistema Único de Saúde), que contém dados secundários de domínio públicos e garante total sigilo acerca das informações pessoais dos indivíduos, como previsto pelo artigo $5^{\circ}$, inciso $\mathrm{X}$, que diz serem invioláveis a intimidade e a honra do paciente (CONSTITUIÇÃO FEDERAL, 1988).

Foram selecionados para a pesquisa somente adultos na faixa etária de 20 a 59 anos- as outras idades foram excluídas porque estavam a um intervalo de 4 anos cada e as posteriores a 59 anos é devido ao tamanho da amostrade ambos os sexos, que sofreram óbitos no estado do Pará e disponibilizados online de acesso aberto, o que dispensa a necessidade de aprovação em Comitê de Ética em Pesquisa, segundo a Resolução do Conselho Nacional de Saúde (CNS) no 466/12. Também foi utilizada a declaração Garther para o desenvolvimento desse estudo, pois ela delimita as melhores condutas para relatos de estudos que quantificam a saúde de populações e seus determinantes por meio da síntese de informações de variadas fontes tendo sido respeitados do mesmo modo a declaração de helsinque e o código de nuremberg (STEVENS, G.A. et al., 2017).

A coleta de dados, iniciou-se por meio do site do Datasus, em seguida-estatísticas vitais-mortalidade 1996 a 2017, pela CID-10 -classificação internacional de doenças(DATASUS),-abrangência geográfica Pará, foram selecionados períodos de 2007 a 2017, sendo a linha sempre as Regiões de saúde (total de 13): metropolitana I, II e III, Rio Caetés, Marajó I e II, Tocantins, Lago de Tucuruí, Baixo Amazonas, Xingu, Carajás, Tapajós e Araguaia, enquanto as colunas foram atribuídas as variáveis sexo, escolaridade, cor/raça, faixa etária, estado civil e local de ocorrência, a categoria selecionada foi a CID10-(códigos B20:

doença para HIV resultado em doença infecciosa e parasitária, B21:doença para HIV resultado em neoplasias malignas, B22: doença para HIV resultado em outras doenças específicas, B23: doença para HIV resultado em outra doença e B24: doença para HIV nenhuma específica), e o conteúdo designado foi óbitos por ocorrência. A partir disso as tabulações dos dados foram efetuadas no software Excel®.

Para a criação e edição do mapa, foi utilizado o software QGIS® versão 3.10.1. Em seguida foram atribuídos os valores correspondentes a cada região e 
realizado pela pesquisadora a quebra natural (Jenks) que é uma função para substituir o desvio padrão devido aos intervalos dispersos.

\section{RESULTADOS}

A tabela 1, apresenta a série histórica dos casos de óbito por HIV/aids ao longo dos anos.

Tabela 1: distribuição temporal de mortalidade por HIV/AIDS. Pará, Região Norte, Brasil, 2007-2017. (n=5.217)

\begin{tabular}{|c|c|c|c|c|c|c|c|c|c|c|c|c|}
\hline \multirow[t]{2}{*}{ Região de saúde } & \multirow[b]{2}{*}{2007} & \multirow[b]{2}{*}{2008} & \multirow[b]{2}{*}{2009} & \multirow[b]{2}{*}{2010} & \multirow[b]{2}{*}{2011} & \multirow[b]{2}{*}{2012} & \multirow[b]{2}{*}{2013} & \multirow[b]{2}{*}{2014} & \multirow[b]{2}{*}{2015} & \multirow[b]{2}{*}{2016} & \multirow[b]{2}{*}{2017} & \multirow[t]{2}{*}{ total } \\
\hline & & & & & & & & & & & & \\
\hline Araguaia & 7 & 2 & 10 & 15 & 18 & 16 & 25 & 29 & 19 & 22 & 28 & 191 \\
\hline Baixa Amazonas & 9 & 14 & 15 & 15 & 30 & 10 & 25 & 32 & 45 & 34 & 25 & 254 \\
\hline Carajás & 29 & 32 & 39 & 24 & 42 & 44 & 50 & 35 & 61 & 45 & 52 & 453 \\
\hline Lago de Tucuruí & 12 & 6 & 13 & 20 & 8 & 23 & 21 & 22 & 18 & 26 & 33 & 202 \\
\hline Metropolitana I & 240 & 262 & 280 & 293 & 299 & 297 & 345 & 351 & 345 & 365 & 349 & 3426 \\
\hline Metropolitana II & 1 & 1 & 0 & 2 & 4 & 1 & 4 & 3 & 9 & 6 & 8 & 39 \\
\hline Metropolitana III & 3 & 3 & 12 & 16 & 17 & 24 & 29 & 28 & 26 & 34 & 34 & 226 \\
\hline Rio Caetés & 5 & 12 & 5 & 20 & 9 & 11 & 15 & 27 & 24 & 25 & 22 & 175 \\
\hline Tapajós & 5 & 2 & 4 & 12 & 10 & 13 & 6 & 9 & 11 & 10 & 8 & 90 \\
\hline Xingu & 3 & 2 & 8 & 3 & 7 & 5 & 8 & 10 & 9 & 24 & 14 & 93 \\
\hline Marajó I & 1 & 1 & 1 & 0 & 1 & 4 & 0 & 1 & 0 & 1 & 3 & 13 \\
\hline Marajó II & 0 & 0 & 0 & 1 & 2 & 0 & 1 & 1 & 2 & 2 & 5 & 14 \\
\hline total & 317 & 344 & 389 & 426 & 451 & 450 & 535 & 550 & 573 & 598 & 584 & 5217 \\
\hline
\end{tabular}

Fonte: dados da pesquisa, DATASUS, 2020.

Percebe-se um aumento de 4.900 casos no estado entre os anos de 2007 a 2017. Desde 2007, apresentando 317 casos, houve um crescimento progressivo até 2011 e tendo algumas das regiões nenhum caso registrado. No ano de 2016 foram registrados 598 mortes, sendo considerado o período mais expressivo. A região Metropolitana I é a que mais se destaca, de início apresentou 240 óbitos, tendo em 2016 o seu pico com 365 casos e representa um total de 3.426 indivíduos, seguida da região de Carajás que teve um índice maior em 2015 com 61 casos e contando com um total de 453 pessoas e seu menor número correspondeu a 29 mortes no ano de 2007. O terceiro lugar é ocupado pelo Baixo Amazonas com 254 mortes na sua totalidade, em 2007 foram registrados 9 óbitos, e em 2015 registrouse 45 casos. Por fim, a região com menor mortalidade foi Marajó I com 13 notificações, tendo nos anos de 2010 e 2015 nenhum registro.

Na figura 1, tem-se o mapa do Pará dividido entre as regiões e a quantidade de casos delimitados em intervalos, tendo sido usado os totais da tabela acima sem a distinção de sexo ou local de ocorrência. Constata-se 13 regiões, que se situam nos intervalos de 13-3.426 casos, onde as regiões mais escuras apresentam maior número de casos e as mais claras o menor índice.

Ao logo da série histórica de 11 anos, houve um aumento significativo em todas as áreas. A região Metropolitana I tem mais casos de mortalidade que as outras regiões, apresentando 3.426 óbitos, seguida de Carajás com 453, Baixo Amazonas com 254 e Metropolitana III com 226 mortes, as regiões com menos mortalidade foram Marajó I e II com 13 e 14 casos respectivamente, mesmo estando localizadas próximas a regiões com maior número registrados.

A tabela 2, apresenta as características dos adultos que evoluíram a óbito devido HIV/AIDS de acordo com o sexo, faixa etária, escolaridade, cor/raça e estado civil. 
Figura 1: Distribuição dos casos de óbito por regiões paraenses. Pará, Região Norte, Brasil, 2007-2017. (n=5.217).

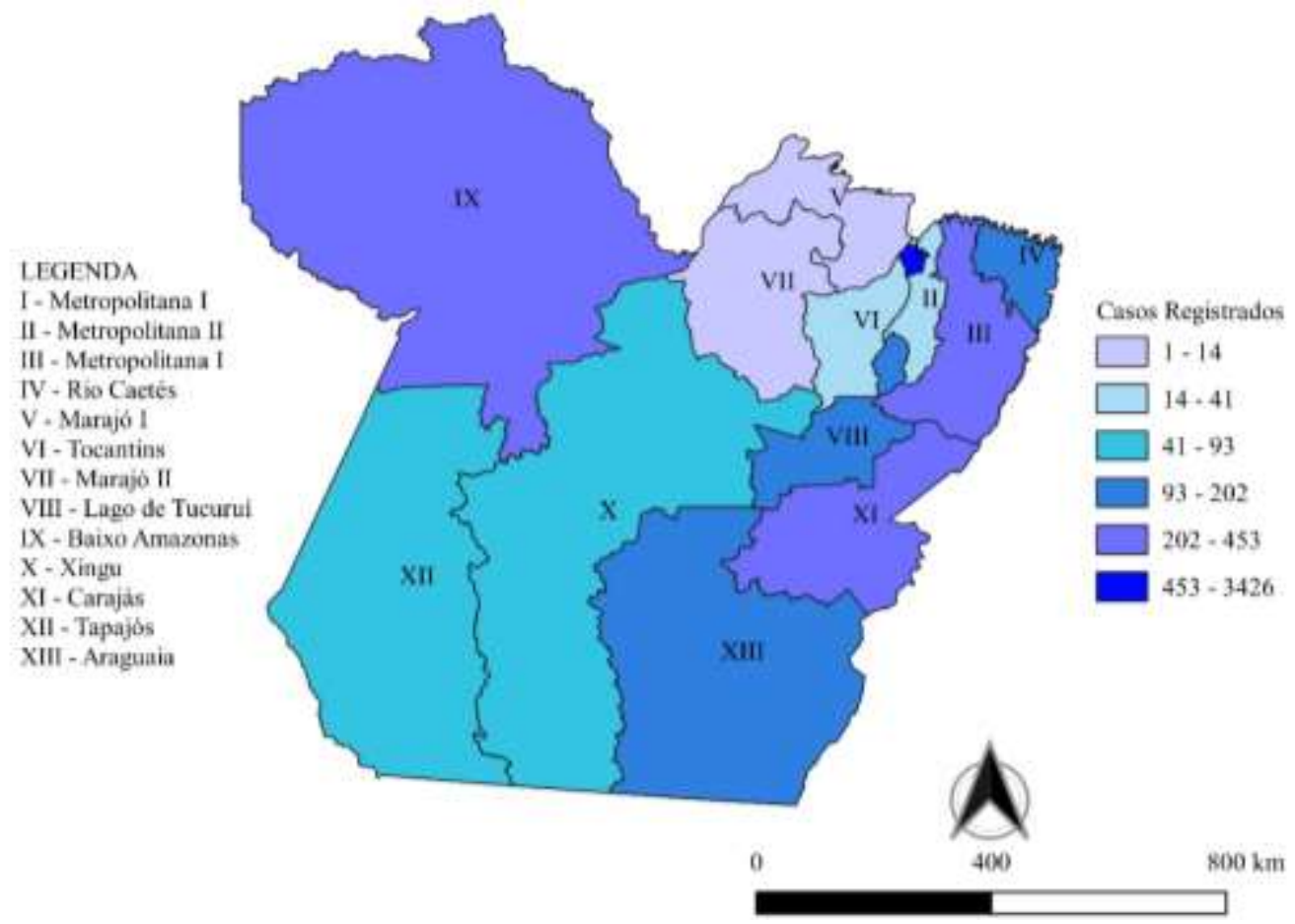

Elaboração: elaborado pela própria autora, 2020.

Tabela 2: Características dos óbitos de adultos por HIV/AIDS de acordo com o sexo. Pará, Região Norte, Brasil, 2007-2017. (n=5.217).

\begin{tabular}{|c|c|c|c|c|c|c|}
\hline \multirow[t]{2}{*}{ Características } & & \multicolumn{2}{|c|}{ Masculino } & \multicolumn{2}{|c|}{ Feminino } & \multirow[t]{2}{*}{ Total } \\
\hline & & $\mathbf{N}$ & $\%$ & $\mathbf{N}$ & $\%$ & \\
\hline \multirow{6}{*}{ Faixa etária (anos) } & 20 a 29 & 776 & $22,2 \%$ & 382 & $22,2 \%$ & 1158 \\
\hline & 30 a 39 & 1214 & $34,7 \%$ & 681 & $39,6 \%$ & 1895 \\
\hline & 40 a 49 & 926 & $26,5 \%$ & 456 & $26,5 \%$ & 1382 \\
\hline & 50 a 59 & 580 & $16,6 \%$ & 202 & $11,7 \%$ & 782 \\
\hline & Total & 3496 & $100,0 \%$ & 1721 & $100,0 \%$ & 5217 \\
\hline & Nenhuma & 266 & $7,6 \%$ & 129 & $7,5 \%$ & 395 \\
\hline \multirow{7}{*}{ Tempo de estudo(anos) } & 1 a 3 anos & 755 & $21,6 \%$ & 423 & $24,6 \%$ & 1178 \\
\hline & 4 a 7 anos & 973 & $27,8 \%$ & 586 & $34,0 \%$ & 1559 \\
\hline & 8 a 11 anos & 839 & $24,0 \%$ & 361 & $21,0 \%$ & 1200 \\
\hline & 12 anos e mais & 286 & $8,2 \%$ & 53 & $3,1 \%$ & 339 \\
\hline & Ignorado & 377 & $10,8 \%$ & 169 & $9,8 \%$ & 546 \\
\hline & Total & 3496 & $100,0 \%$ & 1721 & $100,0 \%$ & 5217 \\
\hline & Branca & 464 & $13,3 \%$ & 187 & $10,9 \%$ & 651 \\
\hline \multirow{4}{*}{ Cor/raça } & Preta & 259 & $7,4 \%$ & 148 & $8,6 \%$ & 407 \\
\hline & Amarela & 10 & $0,3 \%$ & 5 & $0,3 \%$ & 15 \\
\hline & Parda & 2713 & $77,6 \%$ & 1353 & $78,6 \%$ & 4066 \\
\hline & Indígena & 5 & $0,1 \%$ & 4 & $0,2 \%$ & 9 \\
\hline \multirow{9}{*}{ Estado civil } & Ignorado & 45 & $1,3 \%$ & 24 & $1,4 \%$ & 69 \\
\hline & Total & 3496 & $100,0 \%$ & 1721 & $100,0 \%$ & 5217 \\
\hline & Solteiro & 2456 & $70,3 \%$ & 1024 & $59,5 \%$ & 3480 \\
\hline & Casado & 453 & $13,0 \%$ & 274 & $15,9 \%$ & 727 \\
\hline & Viúvo & 40 & $1,1 \%$ & 75 & $4,4 \%$ & 115 \\
\hline & Separado judicialmente & 55 & $1,6 \%$ & 46 & $2,7 \%$ & 101 \\
\hline & Outro & 254 & $7,3 \%$ & 196 & $11,4 \%$ & 450 \\
\hline & Ignorado & 238 & $6,8 \%$ & 106 & $6,2 \%$ & 344 \\
\hline & Total & 3496 & $100,0 \%$ & 1721 & $100,0 \%$ & 5217 \\
\hline
\end{tabular}

Fonte: dados da pesquisa, DATASUS, 2020. 
A tabela 2, mostra que o número de casos masculinos foi de 3.496 e femininos de 1.721 no total. Contudo, em qualquer faixa etária como pode se observar a quantidade de óbitos nos homens é maior em relação as mulheres, cerca de duas vezes. Percebe-se um predomínio em pessoas de 30 a 39 anos, em ambos os sexos, o masculino representa 1.214 enquanto o feminino é de 681. Os mais jovens de 20 a 29 anos esse

correspondendo a 1.200 pessoas, a maioria do sexo masculino. Sendo que o menor número (339) foram os que possuíam maior grau de estudo entre 12 anos e mais anos e por fim os registrados com nenhuma escolaridade foi de 395 no geral.

Sobre a cor/raça, a parda registrou a ocorrência de 4.066 pessoas, correspondendo a 77,6\% nos homens num total de 3.496 indivíduos e $78,6 \%$ do sexo feminino de 1.721 mulheres, seguida da branca com 651 pessoas, homens com valor de 464 e 187 mulheres, o menor número ficou entre os indígenas com um total de 9 número foi de 1.158 , e as porcentagens foram iguais em homens e mulheres (22,2\%). Há um declínio da mortalidade em idades mais avançadas, em pessoas de 50 a 59 anos foram registradas 782 mortes, esse foi o menor índice.

No que se refere ao tempo de estudo, a maioria dos casos foram registrados entre 4 a 7 anos com 1.559 indivíduos, sendo 973 homens e 586 mulheres, seguido de $8 \quad$ a $11 \quad$ anos sujeitos, seguido da amarela com 15 casos juntando os sexos.

Por fim o estado civil dos mais acometidos ficou sendo os declarados solteiros, com $70,3 \%$ dos homens e $59,5 \%$ no sexo feminino totalizando 3.480 pessoas, os casados representaram 727 no geral, sendo 453 homens e 274 mulheres, em $3^{\circ}$ lugar foram os que não se encaixavam em nenhum grupo, já os separados judicialmente correspondem a 101 cidadãos correspondendo ao menor índice.

Em seguida, na tabela 3, tem-se o número de óbitos de acordo com o registro do local de ocorrência

Tabela 3: Local de ocorrência do óbito por HIV nas 13 Regiões de saúde paraenses de acordo com o sexo. Estado do Pará, Região Norte, Brasil, $2007-2017$. $(\mathrm{n}=5.217)$.

\begin{tabular}{|c|c|c|c|c|c|c|c|c|c|}
\hline \multirow{2}{*}{ Região de saúde } & \multicolumn{4}{|c|}{ Local de ocorrência } & \multicolumn{2}{|c|}{ Masculino } & \multicolumn{2}{|c|}{ Feminino } & \multirow[t]{2}{*}{ Total } \\
\hline & Hospital & Domicílio & Via pública & Outros & $\mathbf{N}$ & $\%$ & $\mathbf{N}$ & $\%$ & \\
\hline Araguaia & 172 & 15 & 2 & 2 & 118 & $3,4 \%$ & 73 & $4,2 \%$ & 191 \\
\hline Baixa Amazonas & 232 & 19 & 0 & 3 & 177 & $5,1 \%$ & 77 & $4,5 \%$ & 254 \\
\hline Carajás & 393 & 56 & 2 & 2 & 280 & $8,0 \%$ & 173 & $10,1 \%$ & 453 \\
\hline Lago de Tucuruí & 188 & 10 & 1 & 3 & 115 & $3,3 \%$ & 87 & $5,1 \%$ & 202 \\
\hline Metropolitana I & 3185 & 167 & 4 & 70 & 2360 & $67,5 \%$ & 1066 & $61,9 \%$ & 3426 \\
\hline Metropolitana II & 16 & 21 & 1 & 1 & 24 & $0,7 \%$ & 15 & $0,9 \%$ & 39 \\
\hline Metropolitana III & 159 & 52 & 0 & 15 & 141 & $4,0 \%$ & 85 & $4,9 \%$ & 226 \\
\hline Rio Caetés & 134 & 35 & 0 & 6 & 122 & $3,5 \%$ & 53 & $3,1 \%$ & 175 \\
\hline Tapajós & 83 & 4 & 2 & 1 & 59 & $1,7 \%$ & 31 & $1,8 \%$ & 90 \\
\hline Tocantins & 30 & 10 & 1 & 0 & 23 & $0,7 \%$ & 18 & $1,0 \%$ & 41 \\
\hline Xingu & 90 & 1 & 0 & 2 & 63 & $1,8 \%$ & 30 & $1,7 \%$ & 93 \\
\hline Marajó I & 0 & 7 & 0 & 6 & 5 & $0,1 \%$ & 8 & $0,5 \%$ & 13 \\
\hline Marajó II & 14 & 0 & 0 & 0 & 9 & $0,3 \%$ & 5 & $0,3 \%$ & 14 \\
\hline TOTAL & 4696 & 397 & 13 & 111 & 3496 & $100,0 \%$ & 1721 & $100,0 \%$ & 5217 \\
\hline
\end{tabular}

Fonte: DATASUS, 2020.

A tabela 3, mostra que o ambiente hospitalar liderou o registro de óbitos com 4.696 casos. A região Metropolitana I com 3.185 casos foi a que mais se destacou, seguida de Carajás representando 393 óbitos e Baixo Amazonas com 232 mortes, porém Marajó I não teve nenhuma mortalidade. Relacionado ao domicílio, no total houve 397 registros, com predominância da região metropolitana I com 167, em segundo lugar Carajás com 56 pessoas e Metropolitana III com 52 indivíduos. Na via pública teve 13 óbitos registrados, onde o número máximo foi de 4 casos, também na Metropolitana I. A respeito dos outros locais, obteve-se um total de 111 óbitos, com predominância de Metropolitana I e Metropolitana III com 15 casos.

\section{DISCUSSÃO}

Acerca dos resultados, a região Metropolitana I teve uma maior prevalência dos casos de óbito, assim como Carajás e Baixo Amazonas, a primeira região corresponde as cidades de: Ananindeua, Belém, Benevides, Castanhal, Marituba, Santa Bárbara do Pará e Santa Izabel do Pará com uma população de 2.275.032 segundo o último censo de 2010 (IBGE, 2010) com uma população mais elevada que as outras regiões se pode demonstrar que os casos são proporcionais ao contingente populacional.

$\mathrm{Na}$ região de Carajás, há uma população de 735.830 e Baixo Amazonas com 675.510 ( PES, 2016) a partir desses dados fortalece a ideia de população e infecção, entretanto, percebe-se que áreas mais desenvolvidas como a Capital paraense, é onde tem 
uma mortalidade maior mesmo contando com grandes centros de saúde, ou seja, há falhas na promoção da saúde no estado.

Em relação ao sexo, o masculino prevaleceu em todas as variáveis, isso é resultado de vários fatores como por não procurarem os serviços de saúde e quando procuram não seguem os tratamentos recomendados; geralmente, têm medo de descobrir doenças; não se alimentam adequadamente; estão mais susceptíveis à infecção de IST/Aids e não praticam atividade física com regularidade (SECRETARIA DE ATENÇÃO À SAÚDE, 2018).

A faixa etária mais acometida foram os mais jovens, com um decaimento nos mais idosos, porém a mortalidade em idosos, em um trabalho feito por Graciano et al. (2016, p.11), verificou-se crescimento importante no número de casos a partir de 2008 $(\mathrm{n}=1.553)$, e só houve queda no ano de $2014(\mathrm{n}=758)$. Segundo Camargo ( 2016), os óbitos de pessoas de 60 anos e mais correspondiam a pouco mais de $2 \%$ do total em 1996, chegando a 5\% em 2005 e a quase $10 \%$ em 2014. Ou seja, atualmente de dez pessoas que morrem de aids no Brasil uma corresponde a um idoso.

No que diz respeito ao tempo de estudo, nos resultados observou-se uma prevalência de 4 a 7 anos tanto em homens quanto mulheres, porém elas obtiveram uma porcentagem maior. Esse período de estudo que não é muito, pode ser explicado pela alta taxa de evasão escolar, devido ao mau desempenho, comportamento inadequado e por vezes a escola os orientam indiretamente a sair, além de que as reprovações no Brasil são muito altas quando comparadas a de outros países e mais de $35 \%$ dos alunos repetiram pelo menos um ano no ensino fundamental e médio (PIERI, 2018).

Outro grupo que se destacou foram os que estudaram durante 8 a 11 anos, ou seja, há um nível de conhecimento presente nessas pessoas, muito possivelmente acerca do vírus, no entanto, isso não torna a mortalidade menor. Por fim, há os que não tiveram nenhuma escolaridade e os que tiveram um tempo de estudo maior que 12 anos, foram os que apresentaram menos óbitos, fazendo com que não se estabeleça uma relação direta entre tempo de estudo e mortalidade.

Acerca da cor/raça, a parda se mostrou mais evidente, isso se deve as próprias características do brasileiro que é miscigenado. Segundo o ministério da Saúde (2017, p.10-11): “de 2004 a 2013, aumentou o número de óbitos por aids na raça/cor parda". Em 2013, a maior taxa de mortalidade por Aids registrouse em homens pretos (12,5/100 mil hab.), seguidos de brancos (7,9/100 mil hab.) e pardos (7,1/100 mil hab.). Com relação às mulheres, a maior taxa de mortalidade foi observada na raça/cor preta (8,5/100 mil hab.), seguida da parda $(3,7 / 100$ mil hab.) e da branca $(3,5 / 100$ mil hab.)". (SECRETARIA DE VIGILÂNCIA, 2017).

Resultados parecidos foram encontrados em um estudo no Distrito Federal que ao analisar a distribuição dos casos segundo raça/cor, observa-se que aqueles que se autodeclaram pardos representam a maior categoria, tanto para o HIV quanto para aids
(39,7/42,5\% nos homens e 45,8/40,9\% nas mulheres), seguidos por brancos, tanto em homens como em mulheres (BRASIL, 2019).

Em um trabalho realizado na cidade de Óbidos, os resultados foram semelhantes a este trabalho, a raça/cor ficou em parda com 94,4\% para o sexo feminino e $86,7 \%$ para o masculino (ALVARENGA et al., 2017). Por conseguinte, a aids não apresenta diferenciais de evolução e prognóstico entre as diversas raças, indagase, então, como as diferenças socioeconômicas explicariam indicadores de mortalidade por aids tão díspares entre a população branca e a população negra, sendo esta uma doença na qual mais de $90 \%$ dos pacientes são atendidos nos serviços públicos do Sistema Único de Saúde (SUS), que têm por princípios a universalidade e a equidade dos atendimentos (SANTOS, 2016, p.612).

No Brasil, os indicadores de saúde com base na variável raça/cor revelam desigualdades sociais persistentes no país e destacam os grupos mais vulneráveis. A análise destes indicadores oferece ao poder público informações que podem orientar o desenvolvimento de políticas públicas voltadas às diferentes necessidades da população, contribuindo assim para a promoção da igualdade e da equidade no acesso à atenção à saúde (SECRETARIA DE VIGILÂNCIA, 2017).

No que se refere ao estado civil, em primeiro lugar foram os declarados solteiros, isso pode ser em decorrência do preconceito e na dificuldade de se estabelecer relacionamentos afetivossexuais. Em um trabalho acerca do relacionamento antes e após o diagnóstico do HIV numa amostra de 10 participantes, constatou-se que a maioria deles (sete) não mantém, no momento, relacionamento afetivo e nem sexual (seis), o que foi relacionado, por eles, ao diagnóstico de HIV. Entre outro ponto da pesquisa, dois participantes relataram que após o diagnóstico de HIV iniciaram relacionamento estável e, após compartilharem sua condição sorológica, os companheiros os abandonaram. Outros dois participantes expressaram receio em transmitir o vírus para seus parceiros, o que foi um dificultador nos seus relacionamentos depois do diagnóstico (SÁ; SANTOS, 2018).

A posteriore os casados foram o segundo grupo com maior taxa de mortalidade, no estudo não se sabe se a contaminação foi antes ou após o casamento, porém, sabe-se que ao iniciar um relacionamento monogâmico muitos casais deixam de usar o preservativo e acreditam que estão livres de infeções, incluindo o HIV. Num estudo sobre o uso de preservativos, observa-se ainda, que independentemente do sexo e do estado civil, o motivo predominante em relação ao não uso de preservativo com último parceiro eventual é o "não gosta" e o "não aceita" (NOGUEIRA et al., 2018).

Acerca da localidade desses óbitos, os intra hospitalares representaram a maioria. Não foram encontrados dados específicos para mortalidade em soropositivos, porém estudos feitos em outras localidades sobre outras doenças, falam a respeito disso. De acordo com Souza et al., (2015) 11.177 pacientes adultos admitidos no HU-UEL (Hospital 
Universitário de Londrina-Universidade Estadual de Londrina). Destes, 889 (8,0\%) foram diagnosticados com pelo menos um episódio de IRASs (Infecções relacionadas à assistência à saúde), dos quais 341 evoluíram a óbito, refletindo em taxa mortalidade de $38,4 \%$.

Conclui-se então que o estudo do perfil da mortalidade de uma população é indispensável para subsidiar políticas públicas que visem a melhoria das condições de saúde da mesma. O Sistema sobre Informação de Mortalidade (SIM) é uma das ferramentas utilizadas pela vigilância para a análise do perfil de óbitos ocorridos em determinado território, portanto, a completitude das variáveis da Declaração de Óbito (DO) é essencial para a qualidade da informação (VIGILÂNCIA EPIDEMIOLÓGICA, 2018).

Referente as políticas públicas brasileiras para o controle e prevenção do HIV/aids, destaca-se a terapia antirretroviral (TARV), nesse aspecto o Brasil é considerado modelo no tratamento desde 1996 quando houve a introdução no Sistema Único de Saúde (SUS), da TARV como parte da política brasileira (CIOSAK; JÚNIOR, 2018). E também os Centros de Testagem e Aconselhamento (CTA) que realizam ações de diagnóstico e prevenção de doenças sexualmente transmissíveis (PES, 2016).

\section{CONCLUSÃO}

No estado do Pará, a partir dos resultados obtidos, constatou-se um aumento progressivo de óbitos ao longo dos 11 anos. Na região Metropolitana I, onde fica localizada a capital Belém, representou a maior incidência durante todos os anos com um pico em 2016, assim como Carajás e Baixo Amazonas, que são regiões com muitos municípios e um grande porte populacional.

Na região, há o predomínio da mortalidade entre os jovens, principalmente no sexo masculino, e ao longo da série histórica as mulheres também apresentaram um crescimento. A cor/raça parda, representou a maior parte dos casos, isso se deve a miscigenação e de um processo histórico onde os pardos/negros têm menos acesso a hospitais e atendimentos especializados (SILVA, 2017). No que concerne aos locais dessas mortes, os hospitais lideraram, entretanto, ele ainda é o local mais seguro para atender as demandas do paciente, tendo em vista profissionais qualificados e equipamentos para garantir o suporte a vida.

Cabe ressaltar as políticas públicas atuais para o controle do HIV/aids no estado, uma delas é o fornecimento gratuito de medicamentos antirretrovirais que serve para todo o Brasil, mas especificamente há os Serviços Ambulatoriais em HIV/AIDS- SAE presente em 26 municípios. Além do mais há o Programa Saúde e Prevenção nas Escolas-SPE implantado em 49 municípios paraenses e por fim o Centro de Testagem e Aconselhamento- CTA (PES, 2016). Portanto, este trabalho servirá além das informações acerca do estado, como também dos grupos mais vulneráveis, e poderá ser utilizado para pesquisas cujo objetivo seja de aumentar a sobrevida dos soropositivos e lhes garantir uma vida mais saudável e digna.

\section{REFERENCIAS}

ALVARENGA, A. P. A.; NASCIMENTO, V. B.; SANTOS, L. A. Perfil Epidemiológico de Pacientes Soropositivos no Município de Óbidos/PA, entre os anos de 2012 a 2017. Revista Saúde Integrada, v. 12, n. 24 (2019) - issn 2447-7079.

BRASIL. [Constituição (1988)] Constituição da República Federativa do Brasil : texto constitucional promulgado em 5 de outubro de 1988. - Brasília : Senado Federal, Coordenação de Edições Técnicas, 2016. 496 p. ISBN: 978-85-7018-698-0.

BRASIL. Ministério da Saúde, 2017. Disponível em: <http://www.aids.gov.br/pt-br/noticias/brasilregistra-queda-no-numero-de-casos-e-de-mortes-poraids> Acesso em: 28 de março de 2020.

BRASIL. Ministério da Saúde. Conselho Nacional de Pesquisa. Resolução $\mathrm{N}^{\circ}$ 466, de 12 de Dezembro de 2012.

BRASIL. Ministério da Saúde. Política Nacional de Saúde Integral da População Negra : uma política para o SUS. - 3. ed. - Brasília : Editora do Ministério da Saúde, 2017.

BRASIL. Ministério da Saúde. Secretaria de Atenção à Saúde. Departamento de Ações Programáticas Estratégicas. Perfil da Morbimortalidade Masculina no Brasil. Brasília-DF, p.1-52, 2018.

BRASIL. Ministério da Saúde. Secretaria de Vigilância em Saúde. Boletim Epidemiológico HIV/Aids. Brasília, Número Especial, dez. 2019.

BRASIL. Ministério da Saúde. Secretaria de Vigilância em Saúde. Boletim Epidemiológico HIV/Aids. Brasília, v. 48, p. 1-35, 2017.

BRASIL. Ministério da Saúde. Secretaria de Vigilância em Saúde. Departamento de vigilância, Prevenção e Controle das Infecções Sexualmente Transmissíveis, do HIV/Aids e das Hepatites Virais. Boletim Epidemiológico do HIV/Aids. Brasília, DF; 2018.

CAMARGO, A. B. M. Mortalidade por Aids Entre Idosos no Brasil. Foz do Iguaçu/PR, 2016.

CARVALHO, P. P. et al. Fatores associados à adesão à Terapia Antirretroviral em adultos: Revisão Integrativa de Literatura. Ciência \& Saúde Coletiva, 24(7):25432555, 2019.

DEPARTAMENTO DE INFORMÁTICA DO SUS. CID 10 - DATASUS. Disponível em: $<$ http://datasus1.saude.gov.br/sistemas-e- 
aplicativos/cadastros-nacionais/cid-10> Acesso em: 25 de jan. 2020.

GRACIANO, A. R., et al. Panorama Epidemiológico da Aids no Brasil. RESU- Revista educação em Saúde: V4, N1, 2016 - ISSN: 2358-9868.

GUIMARÃES, M. D. C. et al. Mortalidade por HIV/Aids no Brasil, 2000-2015: Motivos para preocupação?. Revista Brasileira de Epidemiologia, v. 20, p. 182-190, 2017.

IBGE. Censo demográfico, 2010 . Disponível em: $<$ https://www.ibge.gov.br/cidades-eestados.html?view=municipio $>$ Acesso em: 18 de março de 2020.

JÚNIOR, S.S.N.; CIOSAK, S.I.Terapia Antirretroviral Para Aiv/aids: o estado da arte antiretroviral. Recife, abr., 2018,12(4):1103-11.

LIMA, R. L. F. C., et al. Estimativas da Incidência e Mortalidade por Vírus da Imunodeficiência Humana e sua Relação com os Indicadores Sociais nos Estados do Brasil. Revista Brasileira de Ciências da Saúde, 2017, 21(2):139-144.

LIRA, M. Sespa realiza ação alusiva ao Dia Mundial de Luta Contra a AIDS. Disponível em: <http://www.saude.pa.gov.br/2019/12/01/sespa-realizaacao-alusiva-ao-dia-mundial-de-luta-contra-a-aids/> Acesso em: 25 de mar. 2020.

NOGUEIRA, F. J. S., et al. Prevenção, Risco e Desejo: Estudo Acerca do Não Uso de Preservativos. Rev Bras Promoç Saúde, Fortaleza, 31(1): 1-8, jan./mar., 2018.

PIERI, R. Retratos da Educação no Brasil. São Paulo, p. 1-38, 2018 .
SÁ, A. A. M.; SANTOS, C. V. M. A Vivência da Sexualidade de Pessoas que Vivem com HIV/Aids. Psicologia: Ciência e Profissão, Out/Dez. 2018 v. 38 $\mathrm{n}^{\circ} 4,773-786$.

SANTOS, N. J. S. Mulher e negra: dupla vulnerabilidade às DST/HIV/aids. Saúde Soc. São Paulo, v.25, n.3, p.602-618, 2016.

Secretaria de Estado e Saúde Pública. Plano Estadual de Saúde do Pará. PES-PA 2016-2019. Belém/PA, 2016.

SILVA, E. L. V. Racismo Institucional e Suas Repercussões ao Acesso à Saúde no Brasil. LondrinaPR, de 04 a 07 de Julho de 2017.

SOUZA, E.S., et al. Mortalidade e Riscos Associados a Infecção Relacionada à Assistência à Saúde. Texto Contexto Enferm, Florianópolis, 2015 Jan-Mar; 24(1): 220-8.

STEVENS GA, ALKEMA L, BLACK RE, BOERMA JT, COLLINS GS, EZZATI M, et al. Diretrizes para o relato preciso e transparente de estimativas de saúde: a Declaração GATHER. Epidemiol. e Serv. saude Rev. do Sist. Unico Saude do Bras. 2017 Jan 1;26(1):215222.

TRINDADE, F. F.; FERNANDES, G. T.; NASCIMENTO, R, H. F.; JABBUR, I. F. G.; CARDOSO, A. S. Perfil epidemiológico e análise de tendência do HIV/AIDS. Health NPEPS, v. 4, n. 1, p. 153-165, 2019.

VIGILÂNCIA EPIDEMIOLÓGICA HOSPITALAR. Hospital Universitário da Universidade Federal do Vale do São Francisco Empresa. Petrolina, p.1-12, 2018. 